\title{
Clustering-based Human Locomotion Parameters for Motion Type Classification
}

\author{
Ramona LUCA \\ Institute of Computer Science, Romanian Academy Iasi Branch, \\ 2 T. Codrescu Street, Iași 700481, Romania, \\ ramona.luca@iit.academiaromana-is.ro
}

\begin{abstract}
The paper proposes a classification method of human locomotion types from video sequences based on motion parameters clustering. A set of motion parameters is semi-automatically extracted from training video sequences that contain three different types of movement: walking, jogging and running. The motion parameters (postural, frequential, and cinematic) are stored in a relational database and statistic parameters such as minimum, maximum, average and standard deviation are computed. Then a K-means clustering is applied on all the statistic parameters combinations and the results are evaluated using the purity measure to determine most significant parameters to be used in classification. Because the number of training video sequences is reduced, the proposed method may be used as a model of classification only. The automatic determination of movement parameters will increase the data collection size and real testing of the classification method.
\end{abstract}

Keywords: human locomotion, video analysis, ontology, K-means.

\section{Introduction}

Applications in various fields such as video games [4], dance education [18], [19], sports [3], medicine (mainly rehabilitation) [11], [21], self-driving cars and robotics [5], [6], [10], and security [1] require an improved understanding and representation of human locomotion.

Various types of human activities are categorized in: gestures, actions, interactions, and group activities [1]. Gestures are elementary movements of a body part, for example "waiving hand". Complex activities composed from multiple gestures organized temporally are actions. Some examples of human actions are walking, running, jumping. Interaction is a human activity that involves two or more persons and/or objects. Group activities are activities that involve a group of persons and/or objects. The movements of body parts at joints are mainly rotational and take place in three reference planes: sagittal, frontal and transversal. These movements are flexion and extension (frontal axis), abduction and adduction (sagittal axis), medial and lateral (transverse axis).

Walking is a human action in which one foot touchdown is followed by the touchdown of the other foot in a continuous pattern [3]. One of the differences between walking and running is that in the running cycle for a period of time both feet are in the air [7]. Jogging is a form of running at a slower pace [15].
The type of human locomotion may be indicative for the type of activity, intentions, attitudes, circumstances perceived - e.g., dangers, and mood of the person. Consequently, the fast detection of the type and specifics of the locomotion may indicate dangerous situations, among others.

The analysis of the human movement is qualitative and quantitative. While the qualitative analysis of human movement is a non-numerically analysis and describes human actions as patterns, the quantitative analysis requires a large amount of measurements of the parameters which describe the human action and a computer to perform numerical calculation [3].

Human locomotion represents a complex kinematics, with all the body parts participating, involving a large degree of freedom. In [17], [18] and [19] the authors have proposed semantics of the human movement. A semantic representation of human motion in combination with a motion recognition algorithm for digital human simulation is presented in [16].

The combined use of fuzzy logic and complex dynamics has been advocated by several groups, for example [8], [9], [22], including in representing and classification of human movements [9], [21].

This article follows the track established by the papers [12], [13], [23]. Compared to those 
preliminary papers and to the literature, this article brings the following contributions: a complete statistical characterization of the movement, with the related database, including all angles between the relevant body parts, the derivation of the membership functions based on the statistics, the automatic clustering of the movement sequences in view of objective determination of the membership functions related to a more detailed analysis of the dynamics, and detection of abnormal movements applications, with relevance in the medical and security fields.

Throughout the paper, the main parameters of the human locomotion, as established in the literature, for example in [24], are taken into account. This study follows the same path by analyzing basic human locomotion and proposing a fuzzy ontology of it, based on video analysis.

The paper organization is as follows. Section 2 presents the video database and the method used to determine the parameters of the locomotion. In Section 3 a K-means algorithm was applied on extracted parameters. In the fourth section are presented the experimental results and the last section concludes the paper.

\section{Human Motion Characteristics}

In this study, it has been used KTH video collection taken over homogeneous backgrounds with a static camera with $25 \mathrm{fps}$ frame rate [20], in which are performed three types of human actions: walking, jogging and running by 25 different persons. The information extracted from video sequences is the following parameters which describe the human locomotion: postural parameters (step length, angles), frequential parameter (frequency) and cinematic parameters (velocity) [12], [13].

An application was developed to facilitate the measurement of these parameters (Figure 1, Figure 2). In the application, the description points of the segments which form the angles and length are manually selected and automatically computed. The measurements are stored in a database (Figure 2). Based on the values of these measurements a statistic is generated. The minimum, maximum, average and standard deviation for all measurement for the first ten subjects from KTH video collection

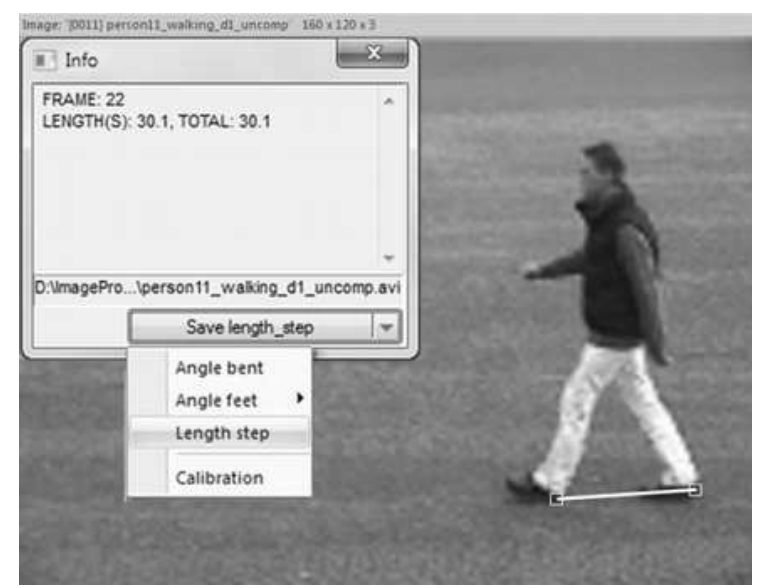

(a)

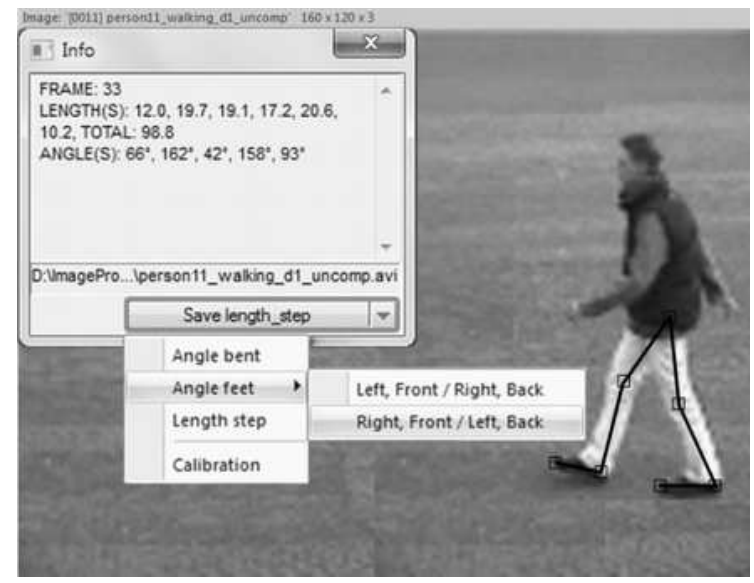

(b)

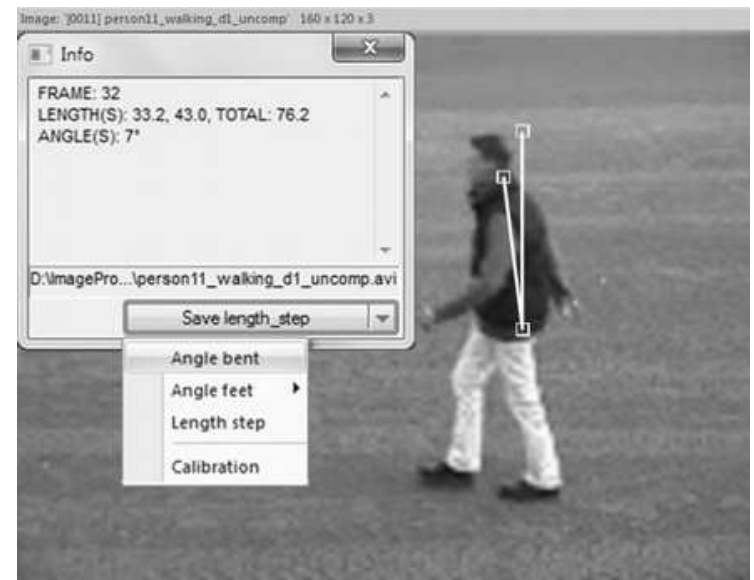

(c)

Figure 1. Parameters capture: (a) step length, (b) feet angles, (c) bent angle.

who perform walking, jogging and running are given in Table 11 from Annex.

The values of the step length parameter are the segment measurements defined by two successive heal touchdown of the ground (Figure1 (a)). For walking, the measurements of step length can be taken in a single frame 


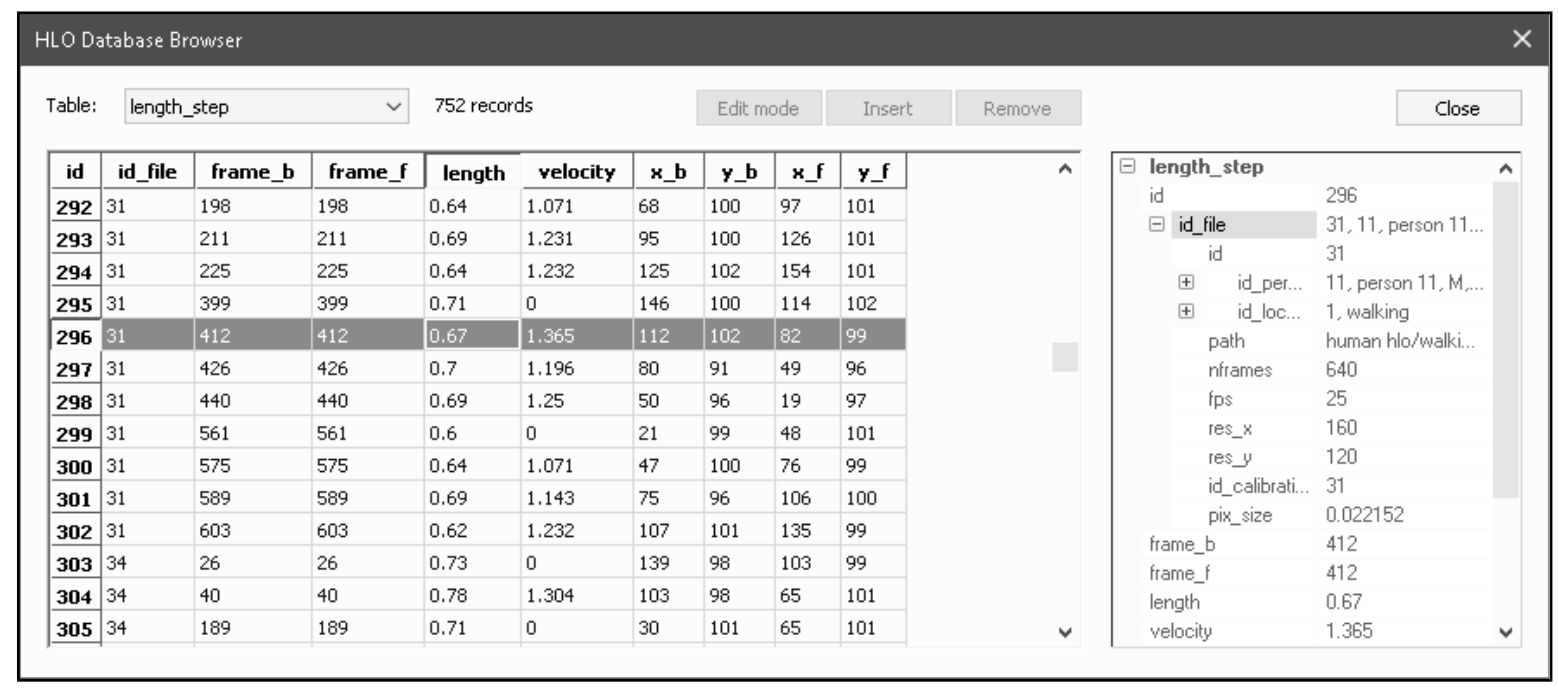

Figure 2. Interface for browsing HLO database (records from step length table)

when both feet are touching the ground. As it was mention before, in the running cycle for a period of time both feet are in the air. There for, the points that determines the step length for jogging and running are in different frames. This can be done only if the camera position is fixed, so the scene position doesn't change from one frame to another. The beginning and the end frames of the step (two successive heel touchdowns) and the frame rate determine the frequency parameter.

Because the video collection doesn't contain information about the height of the persons, it is assumed that men have the average height $1.75 \mathrm{~m}$ and women have the average height $1.65 \mathrm{~m}$. In a calibration step, the pixel size which is required for lengths determinations is computed using person's height.

Having the step length parameter and frequency parameter, the velocity parameter is automatically computed in meter per seconds.

The measured angle parameters are: bent angle, left ankle angle, left knee angle, right ankle angle, right knee angle, and between legs angle. The position of the hand and the angle parameters of the hand were not taken into consideration in this study.

To describe and characterize human actions from images and video sequences using knowledge representation is necessary to define the main concepts related to movements. The main concepts defined in Human Locomotion Ontology (HLO) [12] are related with concepts about:

- joints and parts of the human body;
- frontal, sagittal, and transversal reference planes;

- upper, lower, left, right, front and back relative position defined by the reference planes;

- postural, frequential, and cinematic parameters described above;

- representation of the parameter with linguistic terms, such as low, medium, high or numerical values;

- human actions in this study are walking, jogging and running;

- human condition, such as age or physical condition (normal, disabled).

A relational database was created [13] to store the parameters measurements and it contains also additional important information about the video collection, persons, calibration step, type of locomotion and statistic parameters (minimum, maximum, average and standard deviation) computed by the application.

In [13], average, standard deviation and normal distribution functions for angles parameters and step length were graphically presented. From [12], [13], the most significant parameters are identified and can be used in HLO ontology to define concepts and rules that model human locomotion. The most significant parameters are bent angle, angle between legs, the lowest values of the knee angle, step length, and velocity.

Because some concepts in the HLO ontology are vague, fuzzy datasets were used to annotate the imprecise concepts [13]. By interpreting and analyzing the statistic results, membership 
functions are defined for the fuzzy datasets. For example, the bent angle parameter uses triangular membership functions defined for walking, jogging and running $\mu_{w b}, \mu_{j b}$ and $\mu_{r b}$ as described in (1), (2), (3).

$$
\begin{aligned}
& \mu_{w b}(x)=\left\{\begin{array}{cl}
x / 4 & \text { if } x \in[0,4] \\
1-\frac{x-4}{7} & \text { if } x \in[4,11] \\
0 & \text { otherwise }
\end{array}\right. \\
& \mu_{j b}(x)=\left\{\begin{array}{cl}
x-3 / 8 & \text { if } x \in[3,11] \\
1-\frac{x-11}{8} & \text { if } x \in[11,19] \\
0 & \text { otherwise }
\end{array}\right. \\
& \mu_{r b}(x)=\left\{\begin{array}{cl}
x / 14 & \text { if } x \in[0,14] \\
1-\frac{x-14}{13} & \text { if } x \in[14,27] \\
0 & \text { otherwise }
\end{array}\right.
\end{aligned}
$$

Regarding the knee angle parameters, it was noticed that the lowest value is significant in defining the type of locomotion. Thus, the trapezoidal membership function is used to define the lowest knee angle datatype for walking $(93,93,137,137)$, jogging $(84,84$, $107,107)$ and running $(48,48,102,102)$.

In HLO fuzzy ontology, all the membership functions are defined. For example, the declaration of the trapezoidal membership function of the lowest knee angle for running in our ontology is:

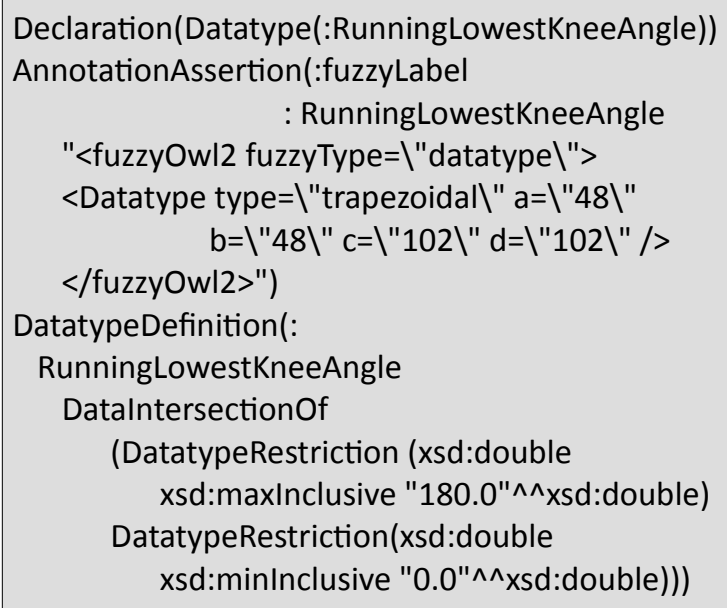

\section{K-means Classification}

For writing the rules that describe the types of locomotion, the K-means clusterization algorithm [2] was applied in the space of the extracted parameters. To find the most significant parameters, the K-means clustering was applied for all their combinations in the training step (Figure 3).

In the training step it was used collected data from the first ten subjects of KTH video collection. The test step uses data from the eleventh subject of KTH video collection (Table 11 from Annex).

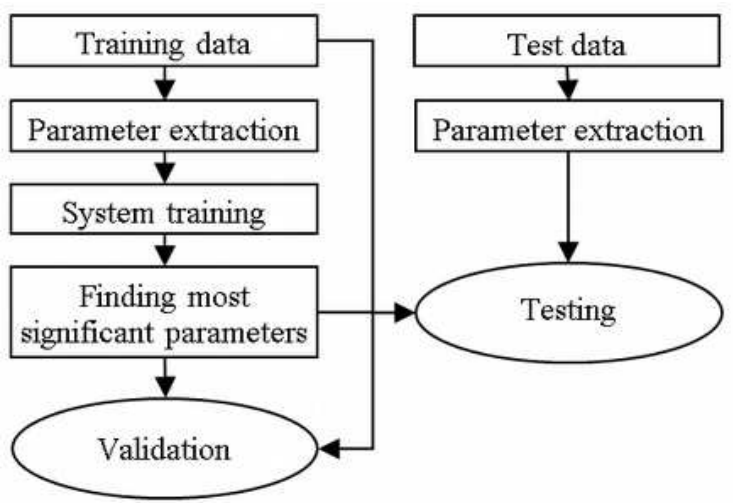

Figure 3. Classification model

Because the velocity parameter sometimes determines alone the locomotion type, two different situations were considered in clustering: with and without using the velocity parameter. To reduce the algorithm's complexity, from each item in the training dataset, only the minimum, maximum and average values of the parameters were considered.

The clustering results were evaluated using the purity measure [24]. Each cluster is assigned to the most frequent class to which the cluster's member belongs.

The purity is computed by evaluating the number of correctly assigned items using:

$$
\text { Purity }=\frac{1}{n} \sum_{i=1}^{n} \text { max }_{\text {cluster }} \cap_{\text {. }} \text { class }_{j} \mid
$$

where $N$ is the number of training data vectors, cluster $_{i}, i=1, N$ are the determined clusters and $\operatorname{class}_{j}, j=1, N$ are the known classes to which the data vectors belong.

The maximum value of purity measure is reached when all items are correctly classified and belong to the proper cluster.

\section{Experimental Results}

The following parameters were used in Kmeans clustering: maximum and average values of bent angle $(\beta)$; minimum values of left knee 
angle $\left(\lambda_{1}\right)$ and right knee angle $\left(\lambda_{\mathrm{r}}\right)$; average and maximum values of between legs angle $(\alpha)$; average and maximum values of step length $(\rho)$; average value of velocity ( $v)$. A human action is represented by parameters vectors.

The Table 1 synthesizes the results of the clusterization for all parameters combinations and it was noticed that the best purity was reached in case of three and four parameter clusterization. The last column shows the number of combinations for which the best purity value was obtained.

Table 1. Synthesis of clusterization results

\begin{tabular}{|c|r|c|}
\hline \# Params & Best purity & \# Best purity \\
\hline 1 & 0.967 & 1 \\
\hline 2 & 0.967 & 6 \\
\hline 3 & 1 & 1 \\
\hline 4 & 1 & 1 \\
\hline 5 & 0.967 & 16 \\
\hline 6 & 0.967 & 6 \\
\hline 7 & 0.967 & 1 \\
\hline 8 & 0.9 & 2 \\
\hline 9 & 0.833 & 1 \\
\hline
\end{tabular}

The maximum value of purity measure obtained when clustering was performed for all parameters combinations is showed in Figure 4.

In case of two parameters clusterization there are six different solutions having the same

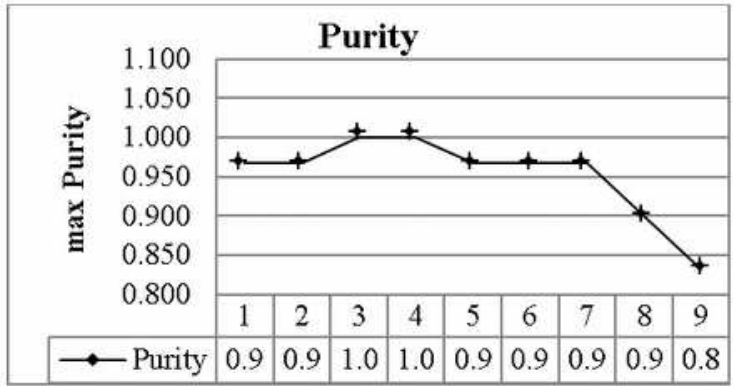

Figure 4. Synthesis of clusterization results.

purity $=0.967$, which means that only one item was wrongly classified.

An example is presented below for the average bent angle and average velocity parameters. In clusterization walking is encoded as class 0 , jogging is class 1 and running is class 2 . The resulted coordinates of centroids for each class in case of two parameter clusterization are presented in Table 2.

Table 2. Coordinates of centroids (two parameters)

\begin{tabular}{|c|c|r|r|}
\hline$\#$ & Class & \multicolumn{1}{c|}{$\boldsymbol{\beta}$ AVG } & \multicolumn{1}{c|}{$\boldsymbol{v}$ AVG } \\
\hline 0 & 0 & 3.230 & 1.460 \\
\hline 1 & 2 & 13.744 & 3.794 \\
\hline 2 & 1 & 7.300 & 2.357 \\
\hline
\end{tabular}

In Figure 5, the three symbols indicate the original class of the parameters pair. The filled symbols indicate the position of centroids for each class. The only incorrectly classified item is marked by a circle in Figure 5.

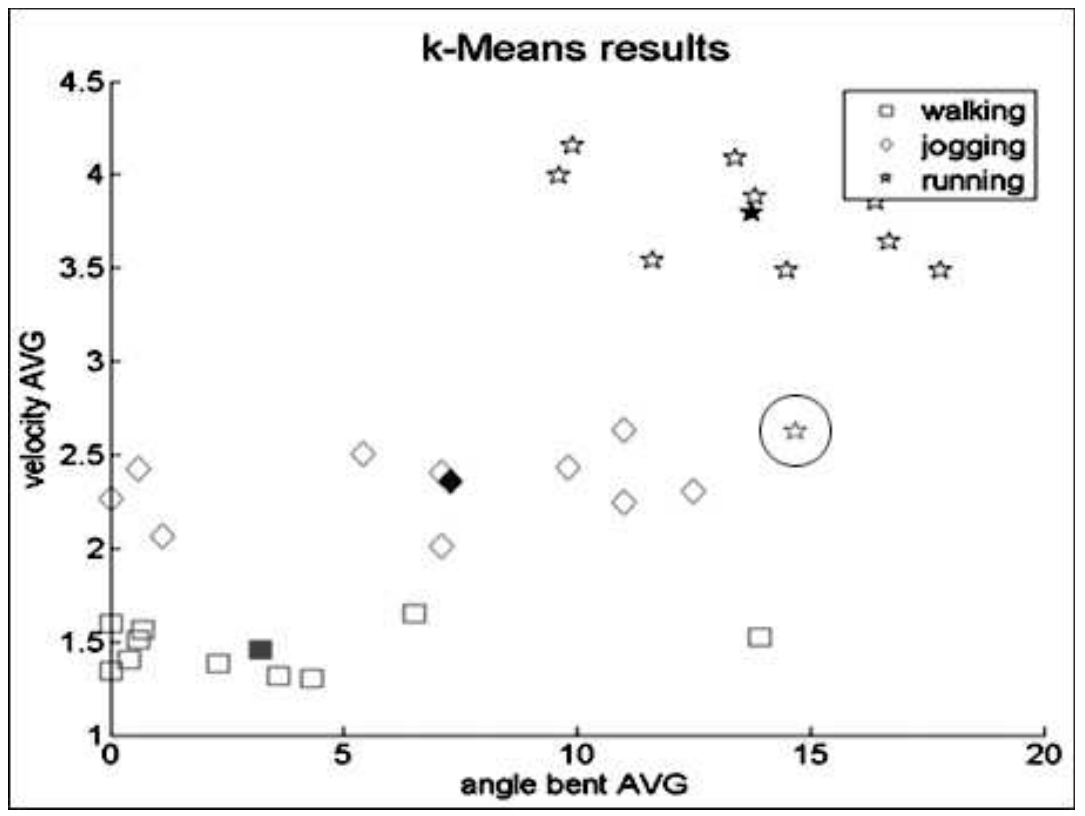

Figure 5. Two parameters - clustering results. 
In Table 3 the validation results for two parameters clusterization are presented. The embossed row is the only item wrongly classified.

Table 3. Validation results in case of two parameters clustering

\begin{tabular}{|c|c|c|c|c|}
\hline ID pers. & Class & \# & $\beta$ AVG & $v \mathrm{AVG}$ \\
\hline 0 & 0 & 0 & 4.3 & 1.301 \\
\hline 0 & 1 & 2 & 7.1 & 2.404 \\
\hline 0 & 2 & 1 & 11.6 & 3.542 \\
\hline 1 & 0 & 0 & 13.9 & 1.527 \\
\hline 1 & 1 & 2 & 12.5 & 2.303 \\
\hline 1 & 2 & 2 & 14.7 & 2.624 \\
\hline 2 & 0 & 0 & 3.6 & 1.317 \\
\hline 2 & 1 & 2 & 11 & 2.636 \\
\hline 2 & 2 & 1 & 13.8 & 3.882 \\
\hline 3 & 0 & 0 & 0.4 & 1.402 \\
\hline 3 & 1 & 2 & 0 & 2.263 \\
\hline 3 & 2 & 1 & 16.7 & 3.645 \\
\hline 4 & 0 & 0 & 2.3 & 1.384 \\
\hline 4 & 1 & 2 & 9.8 & 2.432 \\
\hline 4 & 2 & 1 & 14.5 & 3.486 \\
\hline 5 & 0 & 0 & 6.5 & 1.649 \\
\hline 5 & 1 & 2 & 11 & 2.248 \\
\hline 5 & 2 & 1 & 16.4 & 3.858 \\
\hline 6 & 0 & 0 & 0.7 & 1.566 \\
\hline 6 & 1 & 2 & 5.4 & 2.507 \\
\hline 6 & 2 & 1 & 13.4 & 4.091 \\
\hline 7 & 0 & 0 & 0.6 & 1.512 \\
\hline 7 & 1 & 2 & 7.1 & 2.014 \\
\hline 7 & 2 & 1 & 17.8 & 3.491 \\
\hline 8 & 0 & 0 & 0 & 1.598 \\
\hline 8 & 1 & 2 & 0.6 & 2.428 \\
\hline 8 & 2 & 1 & 9.6 & 3.995 \\
\hline 9 & 0 & 0 & 0 & 1.346 \\
\hline 9 & 1 & 2 & 1.1 & 2.063 \\
\hline 9 & 2 & 1 & 9.9 & 4.153 \\
\hline
\end{tabular}

The Euclidean distances between the parameters vectors extracted (average bent angle, average velocity) for the eleventh person from the test step (Figure 3) and the resulted centroids from Table 2 were computed and the resulted distances are in Table 4. Notice that for this case the eleventh person is classified correctly.

Table 4. Euclidean distances (two parameters)

\begin{tabular}{|l|c|c|c|}
\hline & walking & jogging & running \\
\hline Cluster \#0 & $\mathbf{1 . 2 4 3}$ & 5.733 & 24.352 \\
\hline Cluster \#1 & 12.013 & 5.102 & $\mathbf{1 3 . 6 8 8}$ \\
\hline Cluster \#2 & 5.411 & $\mathbf{1 . 6 4 2}$ & 20.211 \\
\hline
\end{tabular}

For three parameters clusterization, the maximum value of purity measure was obtained for the following combination of parameters: average bent angle, minimum left knee angle and average velocity. The results are depicted in Table 5 and Figure 6.

Table 5. Coordinates of centroids (three parameters)

\begin{tabular}{|c|c|r|r|r|}
\hline$\#$ & Class & \multicolumn{1}{c|}{$\boldsymbol{\beta}$ AVG } & \multicolumn{1}{c|}{$\lambda_{\boldsymbol{l}}$ MIN } & \multicolumn{1}{c|}{$\boldsymbol{v}$ AVG } \\
\hline 0 & 2 & 13.84 & 79.4 & 3.677 \\
\hline 1 & 1 & 6.56 & 95.5 & 2.330 \\
\hline 2 & 0 & 3.23 & 106.1 & 1.460 \\
\hline
\end{tabular}

The Table 6 contains the results of the clusterization in case of three parameters. All items are correctly classified.

Also for this case, the Euclidean distances between parameters vectors average bent angle, minimum left knee angle, average velocity for the eleventh person from the test step (Figure

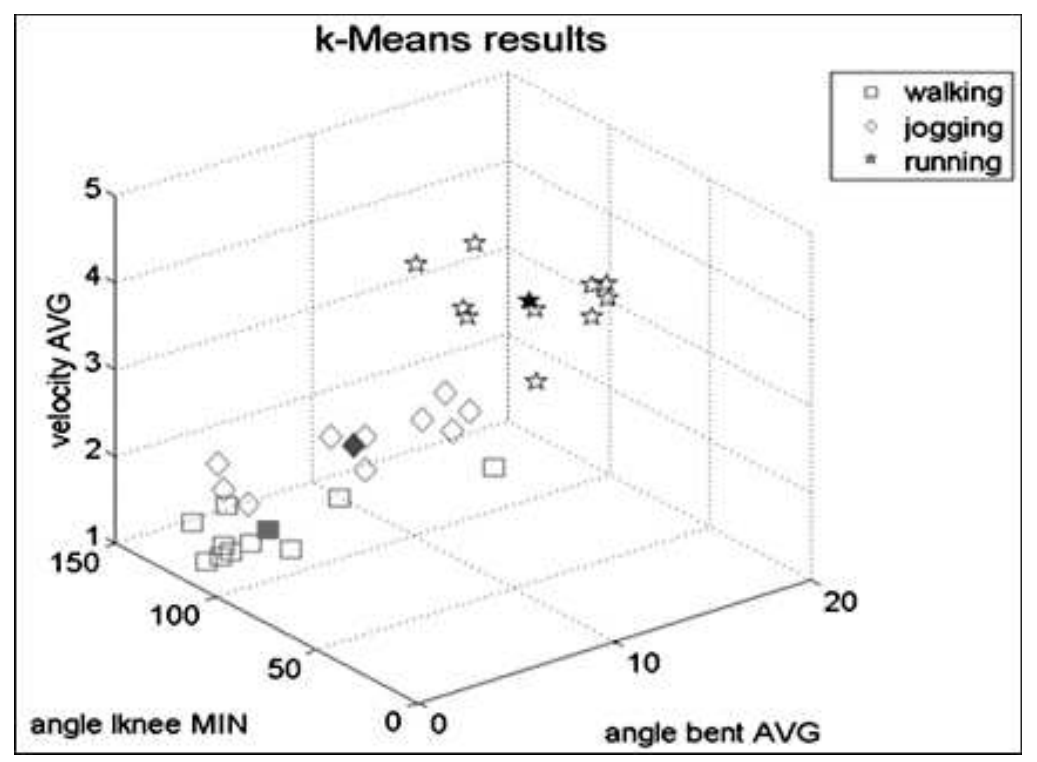

Figure 6. Three parameters - clustering results. 
3) and the centroids (Table 5) were computed. The obtained results are given in Table 7 . Notice that the eleventh person is classified correctly for all classes.

Table 6. Validation results in case of three parameters clustering

\begin{tabular}{|c|c|c|r|r|r|}
\hline $\begin{array}{c}\text { ID } \\
\text { pers. }\end{array}$ & Class & \# & $\boldsymbol{\beta}$ AVG & $\boldsymbol{\lambda}_{\boldsymbol{l}}$ MIN & $\boldsymbol{v}$ AVG \\
\hline 0 & 0 & 2 & 4.3 & 137 & 1.301 \\
\hline 0 & 1 & 1 & 7.1 & 95 & 2.404 \\
\hline 0 & 2 & 0 & 11.6 & 88 & 3.542 \\
\hline 1 & 0 & 2 & 3.9 & 98 & 1.527 \\
\hline 1 & 1 & 1 & 2.5 & 96 & 2.303 \\
\hline 1 & 2 & 0 & 4.7 & 84 & 2.624 \\
\hline 2 & 0 & 2 & 3.6 & 98 & 1.317 \\
\hline 2 & 1 & 1 & 1 & 94 & 2.636 \\
\hline 2 & 2 & 0 & 3.8 & 48 & 3.882 \\
\hline 3 & 0 & 2 & 0.4 & 102 & 1.402 \\
\hline 3 & 1 & 1 & 0 & 84 & 2.263 \\
\hline 3 & 2 & 0 & 6.7 & 68 & 3.645 \\
\hline 4 & 0 & 2 & 2.3 & 105 & 1.384 \\
\hline 4 & 1 & 1 & 9.8 & 93 & 2.432 \\
\hline 4 & 2 & 0 & 14.5 & 83 & 3.486 \\
\hline 5 & 0 & 2 & 6.5 & 102 & 1.649 \\
\hline 5 & 1 & 1 & 1 & 90 & 2.248 \\
\hline 5 & 2 & 0 & 6.4 & 66 & 3.858 \\
\hline 6 & 0 & 2 & 0.7 & 119 & 1.566 \\
\hline 6 & 1 & 1 & 5.4 & 96 & 2.507 \\
\hline 6 & 2 & 0 & 3.4 & 102 & 4.091 \\
\hline 7 & 0 & 2 & 0.6 & 102 & 1.512 \\
\hline 7 & 1 & 1 & 7.1 & 95 & 2.014 \\
\hline 7 & 2 & 0 & 7.8 & 87 & 3.491 \\
\hline 8 & 0 & 2 & 0 & 93 & 1.598 \\
\hline 8 & 1 & 1 & 0.6 & 105 & 2.428 \\
\hline 8 & 2 & 0 & 9.6 & 71 & 3.995 \\
\hline 9 & 0 & 2 & 0 & 105 & 1.346 \\
\hline 9 & 1 & 1 & 1.1 & 107 & 2.063 \\
\hline 9 & 2 & 0 & 9.9 & 97 & 4.153 \\
\hline & & & & & \\
\hline
\end{tabular}

Table 7. Euclidean distances (three parameters)

\begin{tabular}{|l|c|c|c|}
\hline & walking & jogging & running \\
\hline Cluster \#0 & 25.214 & 19.176 & $\mathbf{1 5 . 4 3 6}$ \\
\hline Cluster \#1 & 7.635 & $\mathbf{3 . 3 5 5}$ & 31.416 \\
\hline Cluster \#2 & $\mathbf{4 . 7 3 6}$ & 10.03 & 41.829 \\
\hline
\end{tabular}

In case of clusterization in the four parameters values space, the maximum value of purity was 1 for the following combination of parameters: maximum bent angle, average bent angle, average between legs angle and average velocity (Table 8), also obtained for three parameters. The classification was correctly for this case after applying Euclidean distances Table 9).

Table 8. Coordinates of centroids (four parameters)

\begin{tabular}{|c|c|r|r|r|r|}
\hline \# & Class & $\boldsymbol{\beta}$ MAX & $\boldsymbol{\beta}$ AVG & $\boldsymbol{\alpha}$ AVG & $\boldsymbol{v}$ AVG \\
\hline 0 & 2 & 23.1 & 13.84 & 49.05 & 3.677 \\
\hline 1 & 1 & 10.5 & 6.56 & 29.23 & 2.33 \\
\hline 2 & 0 & 5.5 & 3.23 & 32.94 & 1.46 \\
\hline
\end{tabular}

Table 9. Euclidean distances (four parameters)

\begin{tabular}{|l|c|c|c|}
\hline & walking & jogging & running \\
\hline Cluster \#0 & 47.408 & 49.462 & $\mathbf{2 4 . 9 1 7}$ \\
\hline Cluster \#1 & 11.848 & $\mathbf{3 . 5 2 6}$ & 43.754 \\
\hline Cluster \#2 & $\mathbf{5 . 3 0 6}$ & 8.551 & 45.751 \\
\hline
\end{tabular}

As it was specified, in all cases the best clusterization results were obtained when the velocity was included in the parameters list. We tested the clusterization without considering the velocity parameter. The best value obtained for purity measure was 0.90 for all parameters combinations, which means that three items were classified in a wrong class.

\section{Discussions and Conclusions}

This paper proposes a method to classify walking, jogging and running human actions based on the K-means algorithm. The purpose is to build a system model to recognize different human actions from video sequences using the parameters described in human locomotion ontology. The HLO ontology contains main concepts about human body parts, reference planes, relative position of a body part, parameters and their representation, human actions and conditions. Due the fact that some concepts are vague, fuzzy datasets were used to annotate imprecise concepts in the HLO ontology.

For this study KTH video collection was used which contains various types of locomotion, such as walking, jogging, and running. The extraction of parameters was semi-automatic, which means that the frames and the points that describe segments were manually selected and the values of the parameters were automatically determined. Typically, the sampling corresponds with the frames in which the segments are clearly defined.

As an objective criterion for the most significant parameters selection, the K-means 
algorithm was used. By evaluating the clustering results, two different combinations of three and respectively four parameters for which the purity measure reaches its maximum value on the training dataset were identified.

\section{Acknowledgements}

The reported research and the publication are partly supported by the NATO Grant SPS 984877 "Modeling and Mitigation of Social Disasters Caused by Catastrophes and Terrorism". Also, the research for the second section was supported by the Romanian Academy. The author thanks colleagues H.-N. Teodorescu for comments and suggestions and S.I. Bejinariu for help and support in video processing.

\section{REFERENCES}

1. AGgARWAL, J. K., M. S. RYOO, Human Activity Analysis: A Review, ACM Computing Surveys (CSUR), vol. 43(3), April 2011.

2. ARTHUR, D., S. VASSILVITSKII, Kmeans++: The Advantages of Careful Seeding, SODA '07: Proceedings of the Eighteenth Annual ACM-SIAM Symposium on Discrete Algorithms, 2007, pp. 1027-1035.

3. BARTLETT, R., Introduction to Sports Biomechanics: Analysing Human Movement Patterns, $2^{\text {nd }}$ ed. published in the Taylor \& Francis e-Library, 2007.

4. BRUDERLIN, A., T. W. CALVERT, Goaldirected, Dynamic Animation of Human Walking. Computer Graphics, vol. 23(3), July 1989.

5. COLlins, S., A. RUINA, A Bipedal Walking Robot with Efficient and Human-Like Gait, ICRA, May 2005.

6. COLLINS, S., RUINA, A., TEDRAKE, R., WISSE, M., Efficient Bipedal Robots based on Passive-Dynamic Walkers. Science, vol. 307, 2005, pp. 1082-1085.

7. CROSS, R., Standing, Walking, Running and Jumping on a Force plate, American J. of Physics, vol. 67(4), 1999, pp. 304-309.

8. DE SAlLES, D. C., N. GONCALVES, C. ARMANDO, L. G. MARUJO, Using Fuzzy Logic to Implement Decision Policies in
System Dynamics Models. Exp. Sys. with Applications, vol. 55, 2016, pp. 172-183.

9. JI, X., J. ZHANG, Y. HU, B. RAN, Pedestrian Movement Analysis in Transfer Station Corridor: Velocitybased and Acceleration-based. Physica AStatistical Mechanics and Its Applications, vol. 450, 2016, pp. 416-434.

10. KIM, Y. T., H. C. CHO, J. Y. SEO, H. Y. JEON, G. J. KLIR, Intelligent Path Planning of Two Cooperating Robots based on Fuzzy Logic, Intl. J. General Systems, vol. 31(4), 2002, pp. 359-376.

11. KNUDSON, D., Fundamentals of Biomechanics, Springer, 2007.

12. LUCA, R., H.-N. TEODORESCU, S. I. BEJINARIU, An Ontology of Human Walk for Autonomous Systems, Proc. of 18th International Conference on Systems Theory, Control and Computing, Sinaia, Romania, 2014, pp. 494-499.

13. LUCA, R., Statistical Analysis of Some Parameters Describing Human Locomotion, 5th IEEE Intl. Conference on E-Health and Bioengineering, EHB 2015, Iasi, Romania, 2015, pp. 1-4.

14. MANNING, C. D., P. RAGHAVAN, H. SCHÜTZE, Introduction to Information Retrieval, Cambridge University Press. 2008, pp 356-358, http://nlp.stanford.edu/ IR-book/pdf/16flat.pdf

15. Merriam Webster Dictionary, http://www.merriam-webster.com/dictionary/ jogging, last accessed on 01.06.2016.

16. PINTZOSA, G., N. NIKOLAKISA, K. ALEXOPOULOSA, G. CHRYSSOLOURIS, Motion Parameters Identification for the Authoring of Manual Tasks in Digital Human Simulations: An Approach using Semantic Modelling, Elsevier, 48th CIRP Conference on Manufacturing systems CIRP CMS, 2015.

17. RODRIGUEZ, N. D., R. WIKSTRÖM, J. LILIUS, M. PEGALAJAR CUÉLLAR, M., DELGADO, C. FLORES, Understanding Movement and Interaction: An Ontology for Kinect-Based 3D Depth Sensors, Ubiquitous Computing and Ambient Intelligence. Context-Awareness and Context-Driven Interaction, Lecture Notes 
in Computer Science, vol. 8276, 2013, pp. 254-261.

18. SAAD, S., S. MAHMOUDI, P. MANNEBACK, P., Semantic Analysis of Human Movements in Videos, Proc. ISemantics 2012, 8th Int. Conf. on Semantic Systems, Graz, Austria, 2012, pp. 141-148.

19. SAAD, S., D. DE BEUL, S. MAHMOUDI, P. MANNEBACK, An Ontology for Video Human Movement Representation based on Benesh Notation, International Conference on Multimedia Computing and Systems (ICMCS), 2012.

20. SCHULDT, C., I. LAPTEV, B. CAPUTO, Recognizing Human Actions: A Local SVM Approach, Proceedings of the Pattern Recognition, 17th International Conference on (ICPR'04), vol. 3, 2004, pp. 32-36, www.nada.kth.se/cvap/actions, last accessed on 01.06.2016.

21. TEODORESCU, H.-N., D. MLYNEK, et al., Analysis of Chaotic Movements and Fuzzy Assessment of Hands Tremor in
Rehabilitation. Proceedings KES '98, Knowledge-Based Intelligent Electronic Systems, 1998. Second International Conference on, Adelaide, Australia, vol. 3, 1998, pp. 340-345.

22. TEODORESCU, H. N., A. KANDEL, M. SCHNEIDER, M., Fuzzy Modeling and Dynamics, Fuzzy Sets and Systems, vol. 106(1), 1999, pp. 1-2.

23. TEODORESCU, H.-N., Taxonomy and Procedures for Adaptive Autonomous Systems in Crowded Hybrid Environments, Proceedings of 6th International Conference on Electronics, Computers and Artificial Intelligence, ECAI 2014, Bucharest, Romania.

24. XIANG, Y., J. S. ARORA, K. ABDELMALEK, Physics-based Modeling and Simulation of Human Walking: A Review of Optimization-based and Other Approaches. Structural and Multidisciplinary Optimization, vol. 42(1), 2010, pp. 1-23.

\section{$\underline{\text { Annex }}$}

Table 10. Statistics of the parameters for the first ten persons from KTH video collection

\begin{tabular}{|c|c|c|c|c|c|c|}
\hline Human Locomotion & Parameter Name & $\#$ & Min & Max & Average & Standard Deviation \\
\hline \multirow{8}{*}{ Walking } & Bent Angle & 100 & 0.00 & 18.00 & 3.23 & 4.41 \\
\hline & Left Ankle Angle & 100 & 62.00 & 112.00 & 88.97 & 11.33 \\
\hline & Left Knee Angle & 100 & 93.00 & 180.00 & 151.70 & 23.67 \\
\hline & Right Ankle Angle & 100 & 60.00 & 151.00 & 87.97 & 13.73 \\
\hline & Right Knee Angle & 100 & 84.00 & 180.00 & 148.26 & 25.50 \\
\hline & Between Legs Angle & 100 & 0.00 & 60.00 & 32.94 & 15.72 \\
\hline & Step Length & 352 & 0.59 & 0.92 & 0.72 & 0.06 \\
\hline & Velocity & 249 & 0.91 & 1.86 & 1.29 & 0.17 \\
\hline \multirow{8}{*}{ Jogging } & Bent Angle & 100 & 0.00 & 19.00 & 6.56 & 5.01 \\
\hline & Left Ankle Angle & 100 & 49.00 & 126.00 & 88.46 & 13.55 \\
\hline & Left Knee Angle & 100 & 84.00 & 179.00 & 139.70 & 23.09 \\
\hline & Right Ankle Angle & 100 & 45.00 & 129.00 & 83.75 & 13.71 \\
\hline & Right Knee Angle & 100 & 75.00 & 178.00 & 137.13 & 25.04 \\
\hline & Between Legs Angle & 100 & 1.00 & 60.00 & 29.23 & 13.44 \\
\hline & Step Length & 259 & 0.58 & 1.16 & 0.89 & 0.11 \\
\hline & Velocity & 259 & 1.52 & 2.98 & 2.24 & 0.28 \\
\hline \multirow{8}{*}{ Running } & Bent Angle & 100 & 0.00 & 39.00 & 13.84 & 6.14 \\
\hline & Left Ankle Angle & 100 & 53.00 & 123.00 & 88.77 & 14.67 \\
\hline & Left Knee Angle & 100 & 48.00 & 171.00 & 127.24 & 29.61 \\
\hline & Right Ankle Angle & 100 & 53.00 & 120.00 & 86.32 & 13.95 \\
\hline & Right Knee Angle & 100 & 54.00 & 177.00 & 132.34 & 29.02 \\
\hline & Between Legs Angle & 100 & 0.00 & 89.00 & 49.05 & 19.99 \\
\hline & Step Length & 141 & 0.80 & 1.83 & 1.29 & 0.20 \\
\hline & Velocity & 141 & 2.14 & 7.10 & 3.74 & 0.81 \\
\hline
\end{tabular}


Table 11. Statistics of the parameters for the eleventh person from KTH video collection used for testing

\begin{tabular}{|c|c|c|c|c|c|c|}
\hline Human Locomotion & Parameter Name & \# & Min & Max & Average & Standard Deviation \\
\hline \multirow{8}{*}{ Walking } & Bent Angle & 10 & 0 & 3.81 & 2.013 & 0.983 \\
\hline & Left Ankle Angle & 10 & 59.55 & 100.78 & 85.728 & 12.572 \\
\hline & Left Knee Angle & 10 & 101.53 & 178.67 & 151.994 & 21.89 \\
\hline & Right Ankle Angle & 10 & 63.43 & 95.39 & 81.983 & 9.939 \\
\hline & Right Knee Angle & 10 & 118.19 & 174.49 & 154.886 & 17.405 \\
\hline & Between Legs Angle & 10 & 25.66 & 48.75 & 37.814 & 6.605 \\
\hline & Step Length & 17 & 0.6 & 0.71 & 0.665 & 0.034 \\
\hline & Velocity & 13 & 1.071 & 1.365 & 1.206 & 0.089 \\
\hline \multirow{8}{*}{ Jogging } & Bent Angle & 10 & 6.12 & 11.69 & 8.925 & 1.604 \\
\hline & Left Ankle Angle & 10 & 67.81 & 112.47 & 91.998 & 11.576 \\
\hline & Left Knee Angle & 10 & 97.87 & 171.95 & 145.3 & 21.017 \\
\hline & Right Ankle Angle & 10 & 73.83 & 102.99 & 91.684 & 8.18 \\
\hline & Right Knee Angle & 10 & 90.17 & 166.76 & 133.131 & 26.281 \\
\hline & Between Legs Angle & 10 & 20.84 & 47.74 & 31.549 & 8.872 \\
\hline & Step Length & 16 & 0.69 & 0.89 & 0.808 & 0.049 \\
\hline & Velocity & 16 & 1.725 & 2.472 & 2.119 & 0.191 \\
\hline \multirow{8}{*}{ Running } & Bent Angle & 10 & 21.04 & 32.47 & 27.425 & 3.651 \\
\hline & Left Ankle Angle & 10 & 70.52 & 120.96 & 91.931 & 15.398 \\
\hline & Left Knee Angle & 10 & 72.09 & 157.49 & 120.193 & 27.596 \\
\hline & Right Ankle Angle & 10 & 71.57 & 102.82 & 84.329 & 9.827 \\
\hline & Right Knee Angle & 10 & 47.27 & 152.38 & 112.234 & 34.747 \\
\hline & Between Legs Angle & 10 & 30.46 & 88.35 & 60.739 & 16.348 \\
\hline & Step Length & 6 & 1.17 & 1.6 & 1.407 & 0.142 \\
\hline & Velocity & 6 & 4.056 & 4.444 & 4.217 & 0.118 \\
\hline
\end{tabular}

